\title{
Visible Enantiomer Discrimination via Diphenylalanine-based Chiral Supramolecular Self-assembly on Multiple Platforms
}

Minggao Qin, Yaqian Zhang, Jinying Liu, Chao Xing, Changli Zhao, Xiaoqiu Dou, Chuanliang Feng*

State Key Lab of Metal Matrix Composites, School of Materials Science and Engineering, Shanghai Jiao Tong University, 800 Dongchuan Road, Shanghai, P. R. China, 200240

*Corresponding author

Phone \& Fax: 86 2154747651; E-mail: clfeng@sjtu.edu.cn 

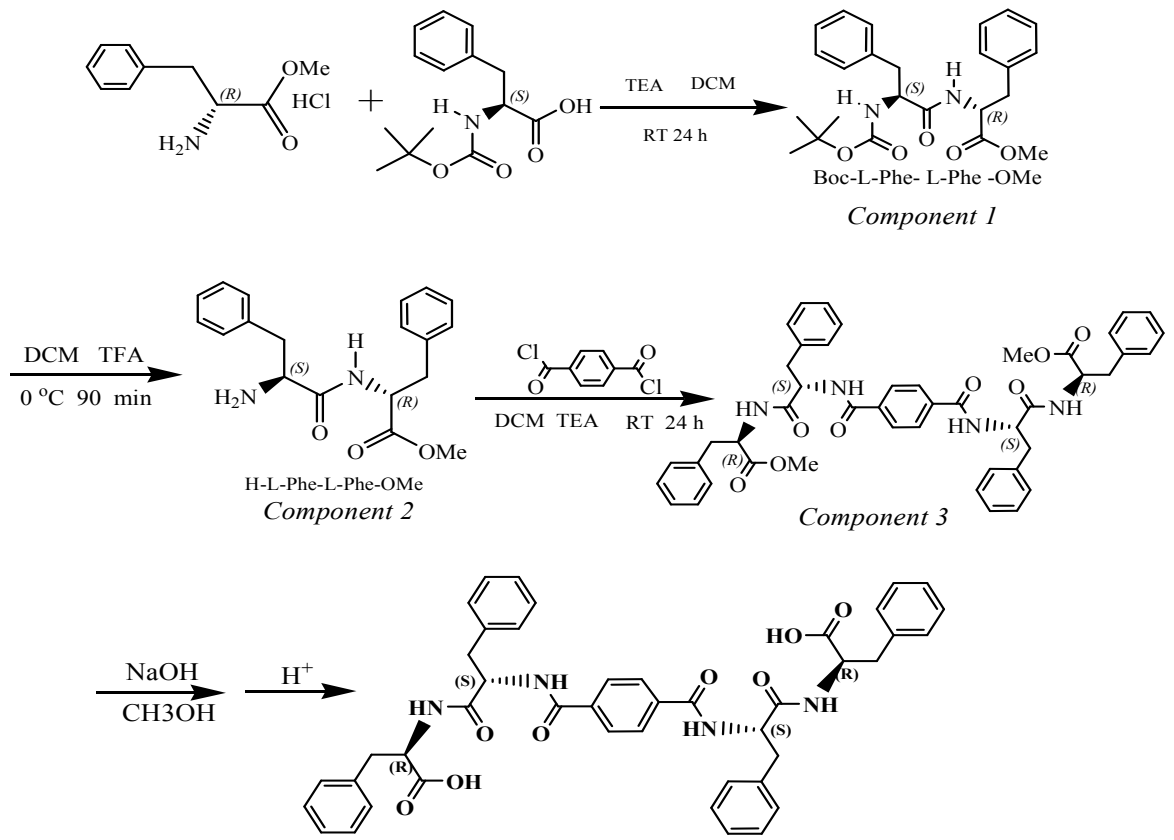

LFDF

Component 4

Scheme S1. Synthesis of gelator LFDF. 


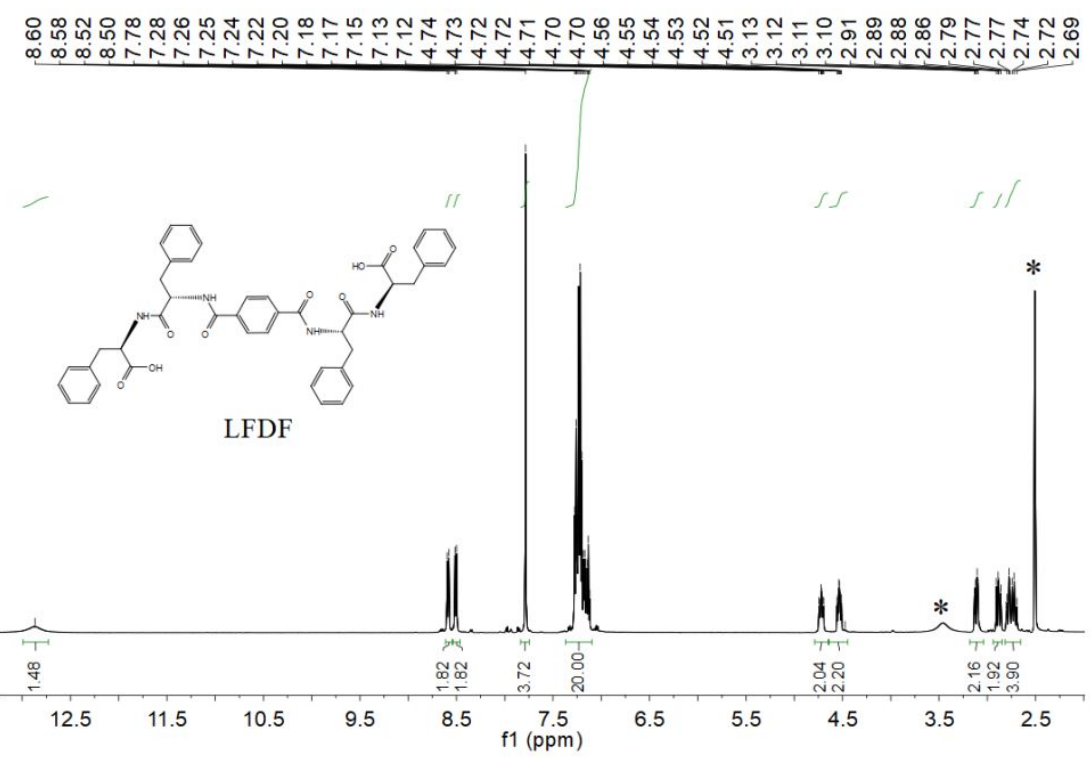

${ }^{1} \mathrm{H}$ NMR (500 MHz, DMSO) $\delta=12.87(\mathrm{~s}, 2 \mathrm{H}), 8.59(\mathrm{~d}, J=8.7,2 \mathrm{H}), 8.52(\mathrm{t}$, $J=9.4,12 \mathrm{H}), 7.78(\mathrm{~s}, 4 \mathrm{H}), 7.35-7.06(\mathrm{~m}, 20 \mathrm{H}), 4.80-4.66(\mathrm{~m}, 2 \mathrm{H}), 4.53(\mathrm{td}$, $J=9.4,4.7,2 \mathrm{H}), 3.11(\mathrm{dd}, J=13.6,4.6,2 \mathrm{H}), 2.88$ (dd, $J=13.6,9.7,2 \mathrm{H}), 2.82$ $2.67(\mathrm{~m}, 4 \mathrm{H})$.

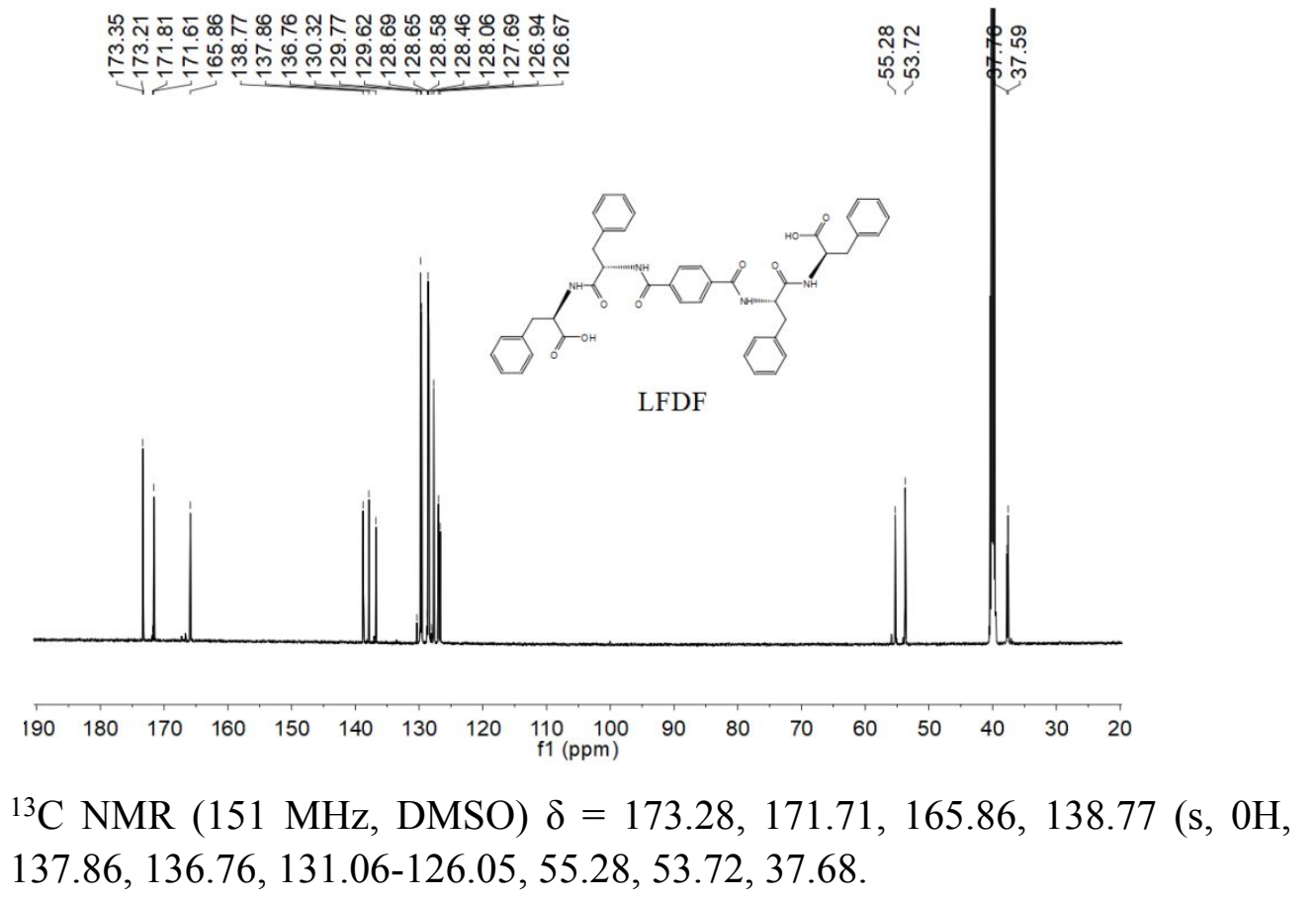

Figure S1. ${ }^{1} \mathrm{H}$ NMR (500 MHz) and ${ }^{13} \mathrm{C}$ NMR (125 MHz) spectra of LFDF in DMSO-d6. 


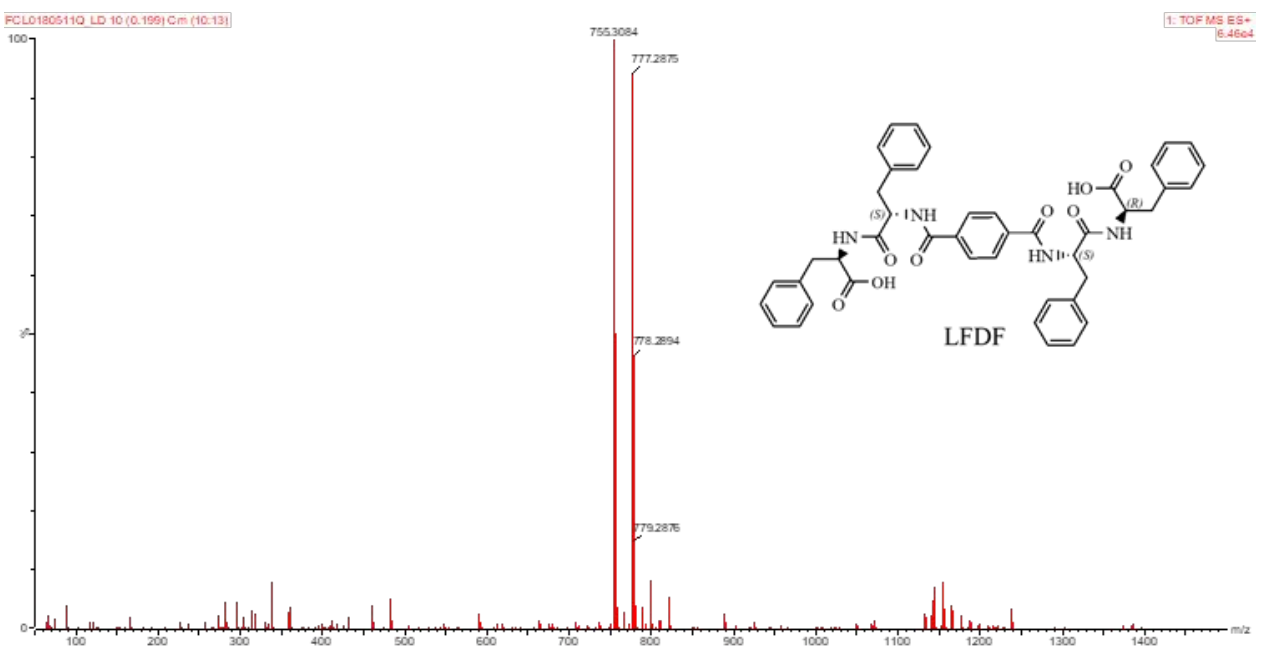

Figure S2. EI-MS spectra of LFDF.

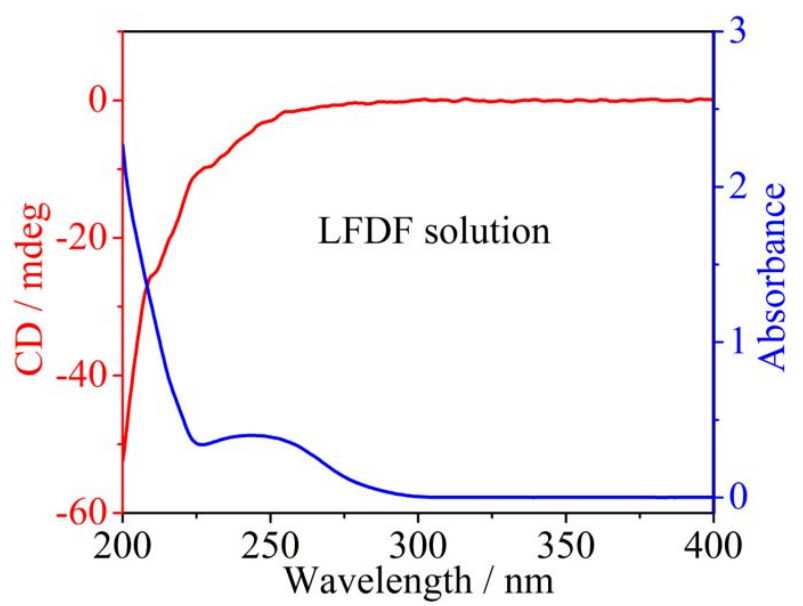

Figure S3. CD and UV-Vis spectra of LFDF molecular state.

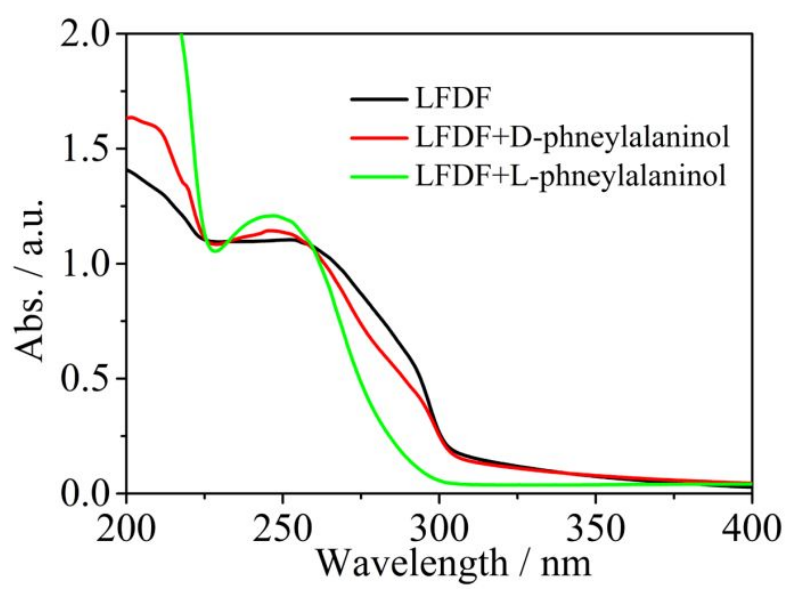

Figure S4. The corresponding absorbance curves of CD spectra. 

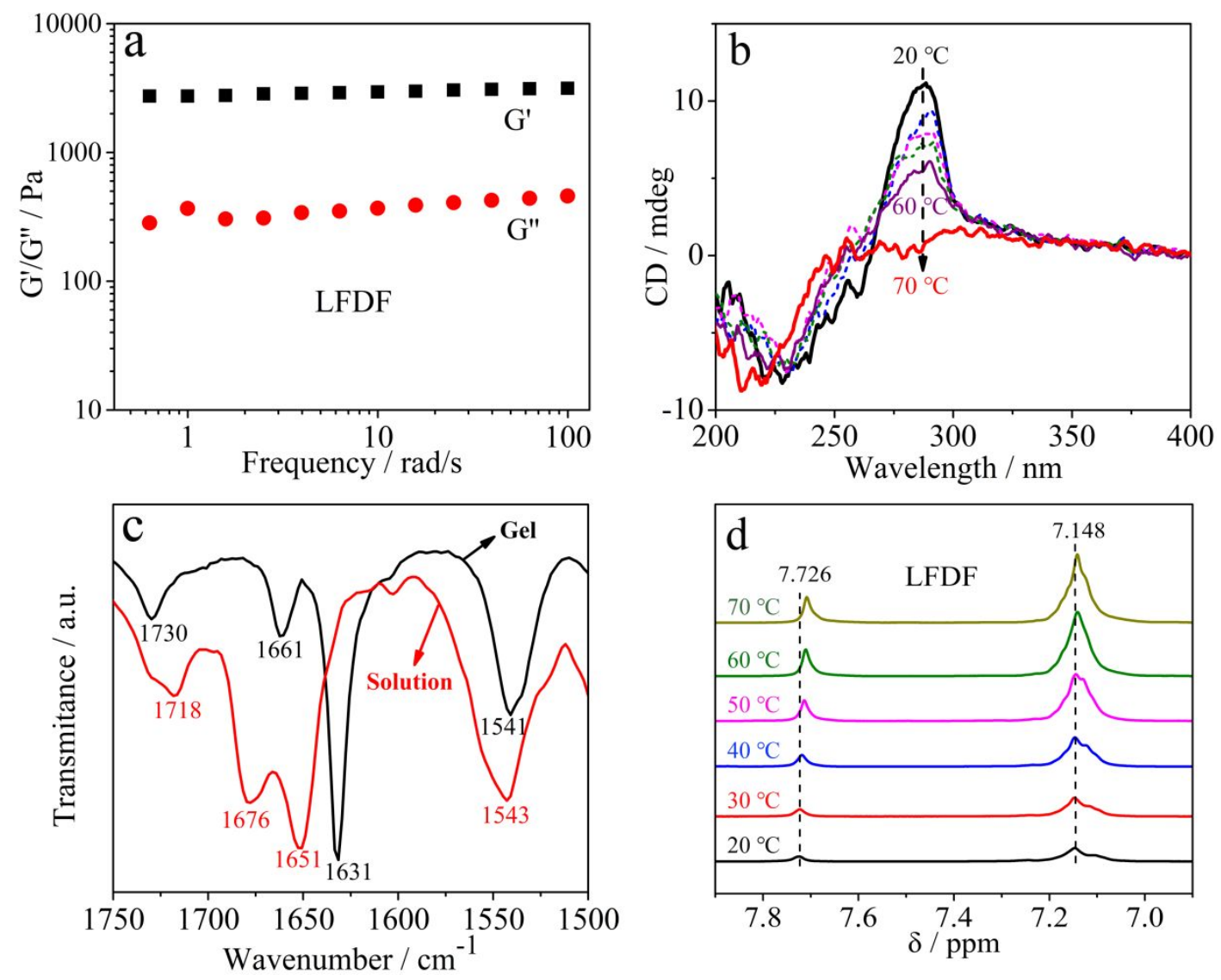

Figure S5. (a) Rheological dynamic frequency sweeps of LFDF gel for monitoring the storage modulus (G') and loss modulus $\left(\mathrm{G}^{\prime \prime}\right)$; (b) Temperature-dependent CD, (c) ATR-FTIR spectra of LFDF solution (red solid line) and supramolecular gel (black solid line), and (d) Temperaturedependent ${ }^{1} \mathrm{H}-\mathrm{NMR}$ spectra of supramolecular gel. 

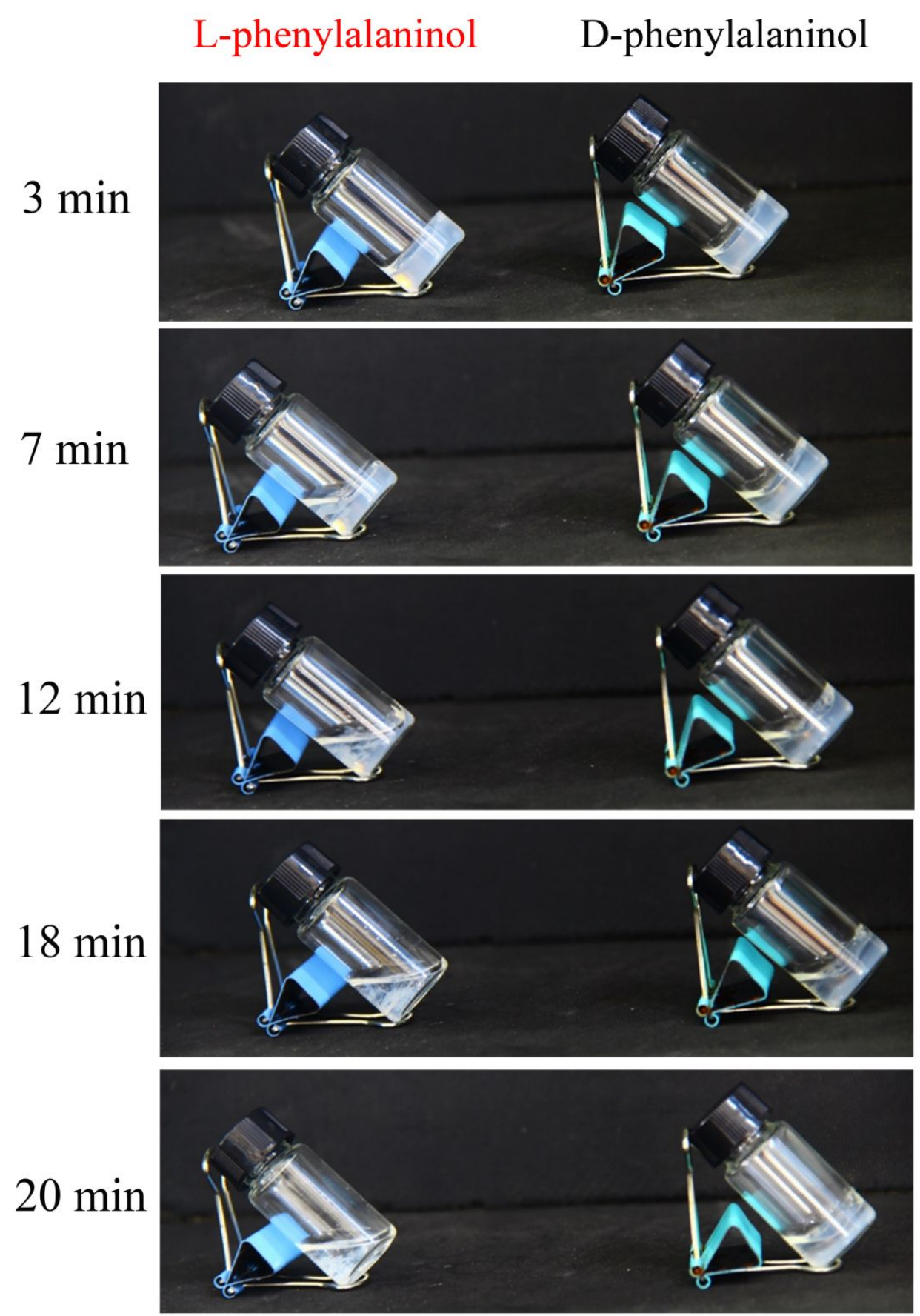

Figure S6. Photos of gel upon adding $L$-phenylalaninol (left) and $D$-phenylalaninol (right) at different time points. 

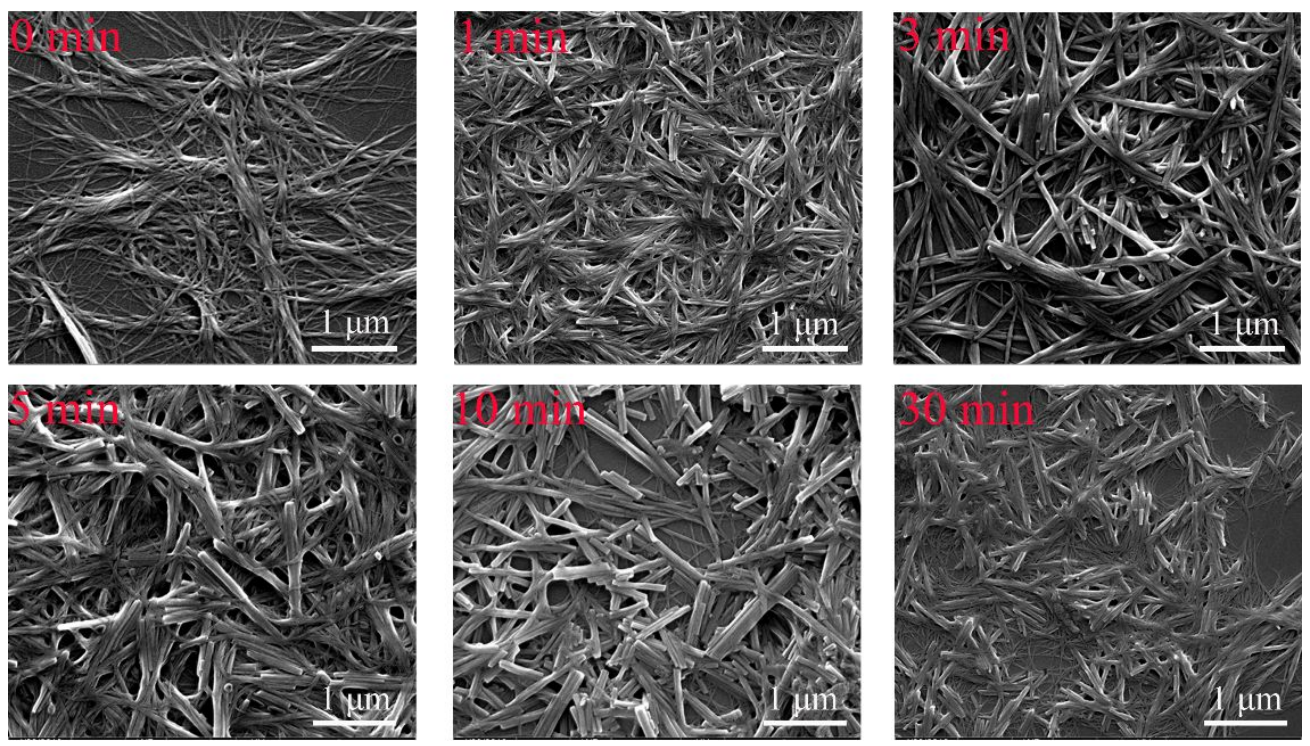

Figure S7. Time-dependent SEM images to visualize the nanostructure changes upon adding $L$ phenylalaninol.
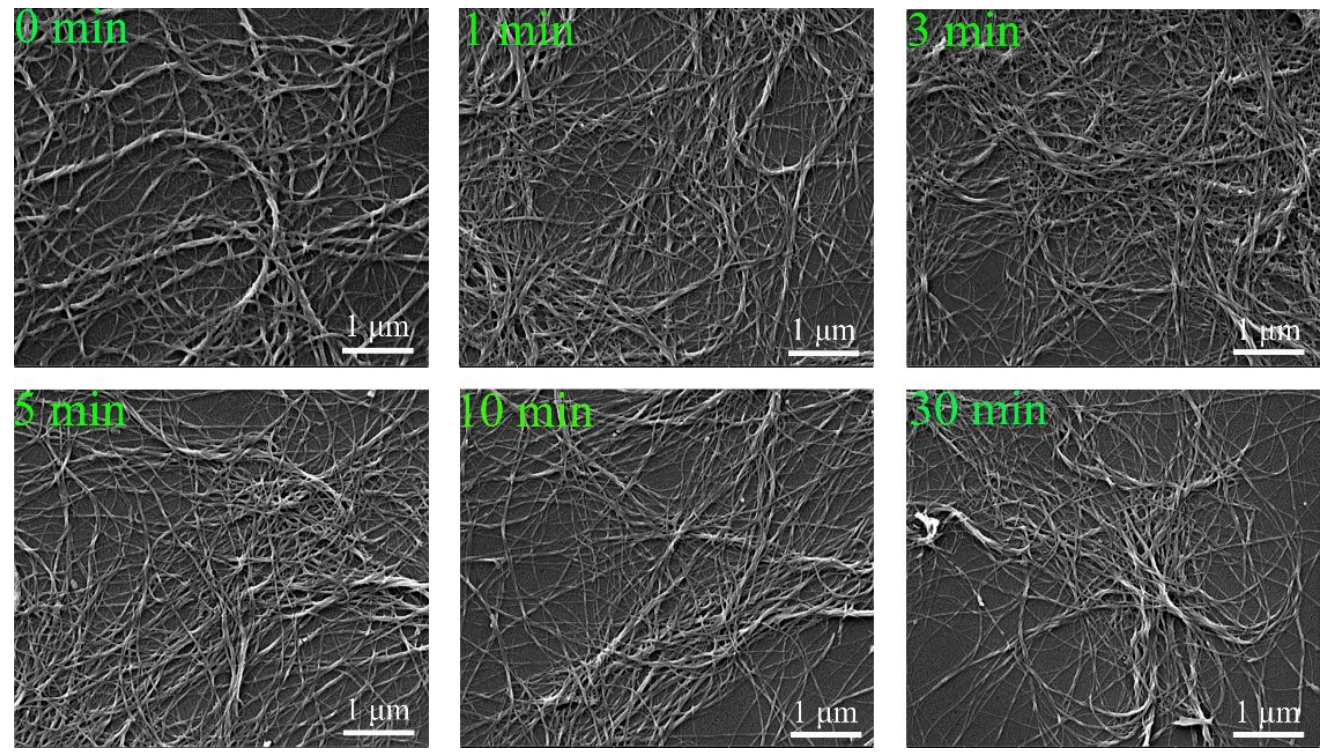

Figure S8. Time-dependent SEM images to visualize the nanostructure changes upon adding $D$ phenylalaninol. 


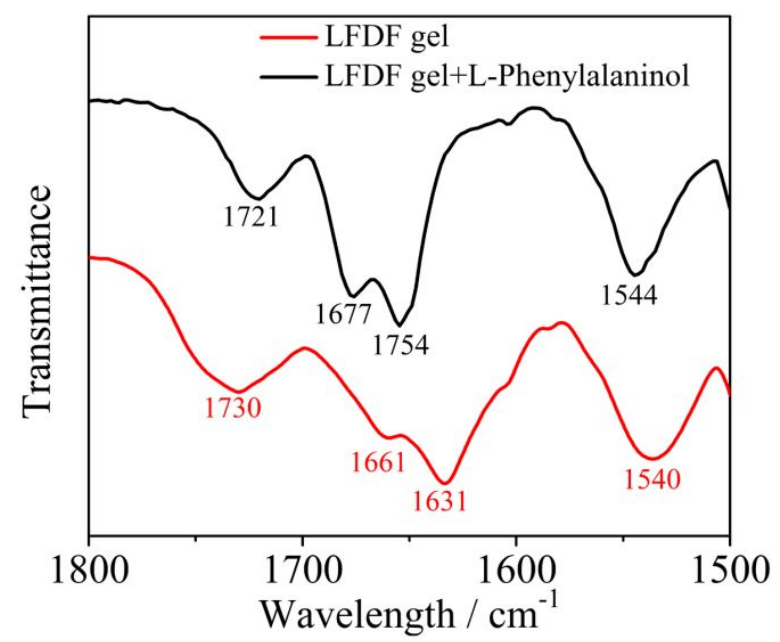

Figure S9. The FTIR spectra before and after adding $L$-phenylalaninol to supramolecular LFDF gel.

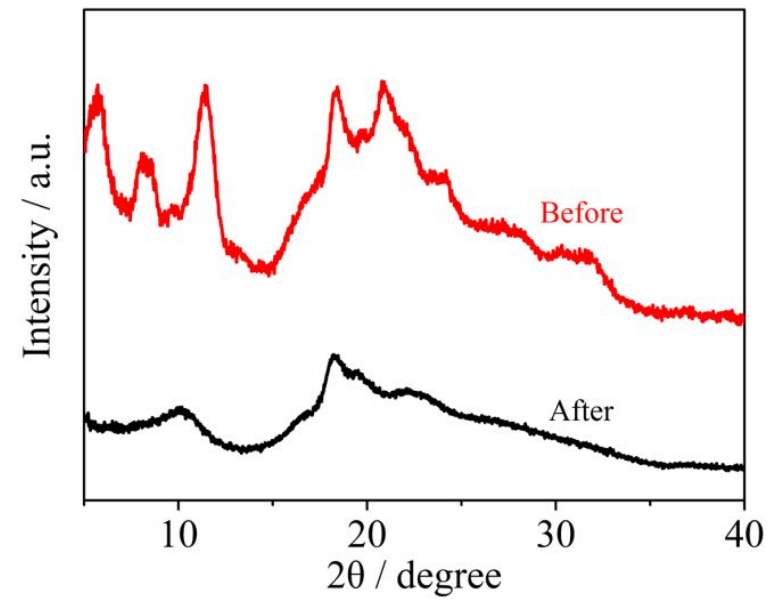

Figure S10. The corresponding XRD before and after adding $L$-phenylalaninol to supramolecular LFDF gel. 


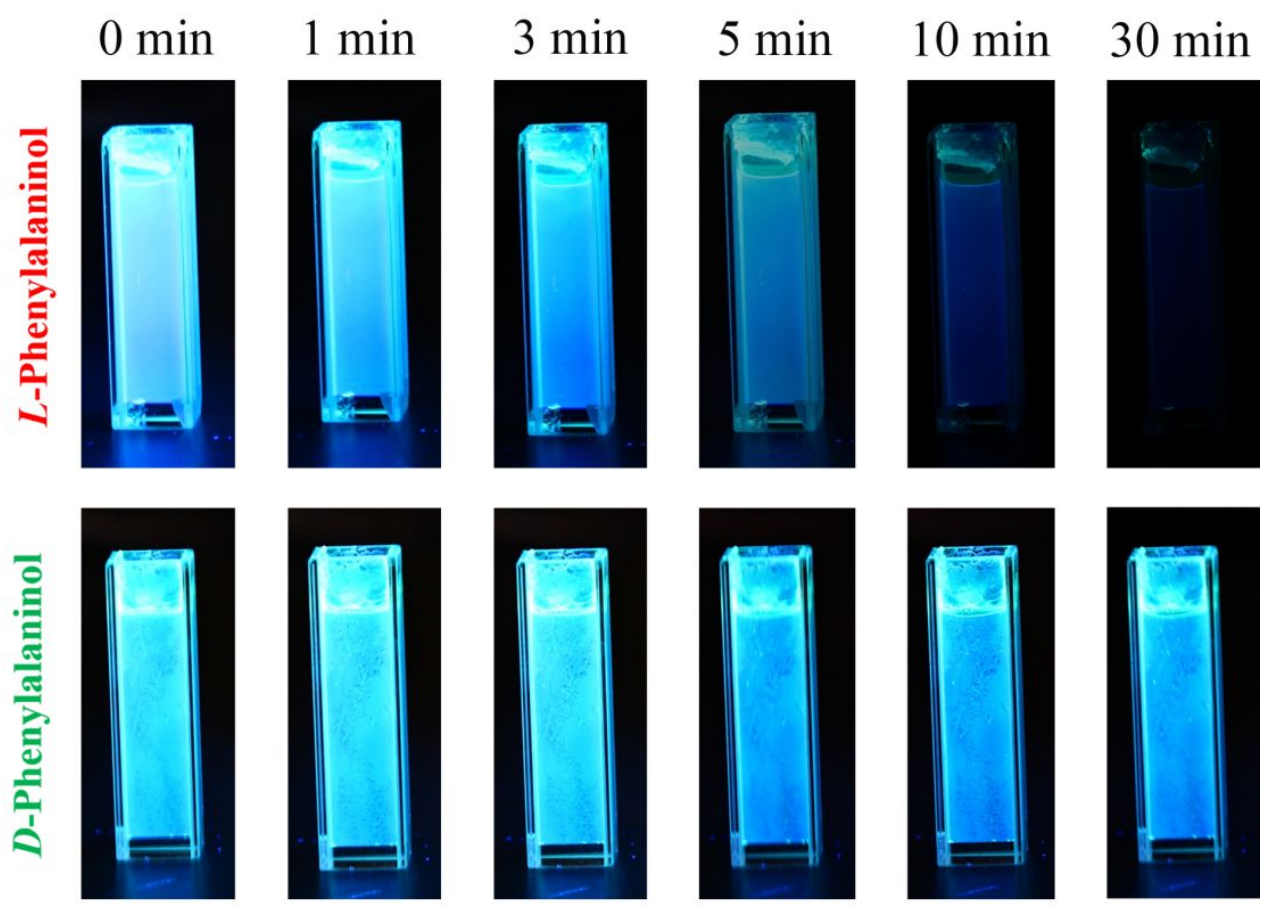

Figure S11. The changes of the blue-green luminescence of ThT-LFDF xerogel in the presence of $L$-phenylalaninol (up) and $D$-phenylalaninol (down) at different time points were clearly visible under UV light.
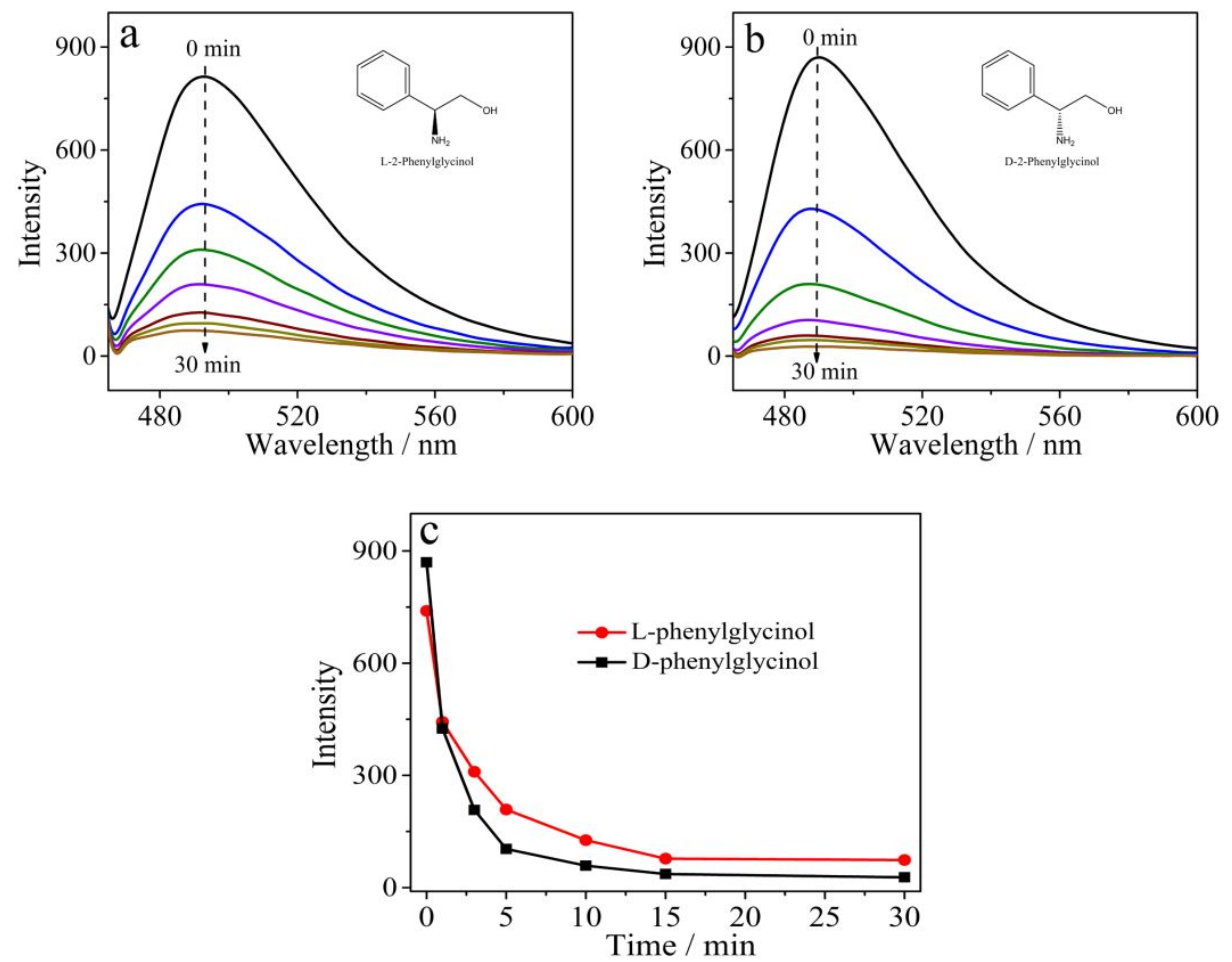

Figure S12. The fluorescence spectra of ThT-LFDF xerogel in the presence of $L$-phenylglycinol (a) and $D$-phenylglycinol (b), and the corresponding plots of fluorescence intensity at $488 \mathrm{~nm}$ (c). 

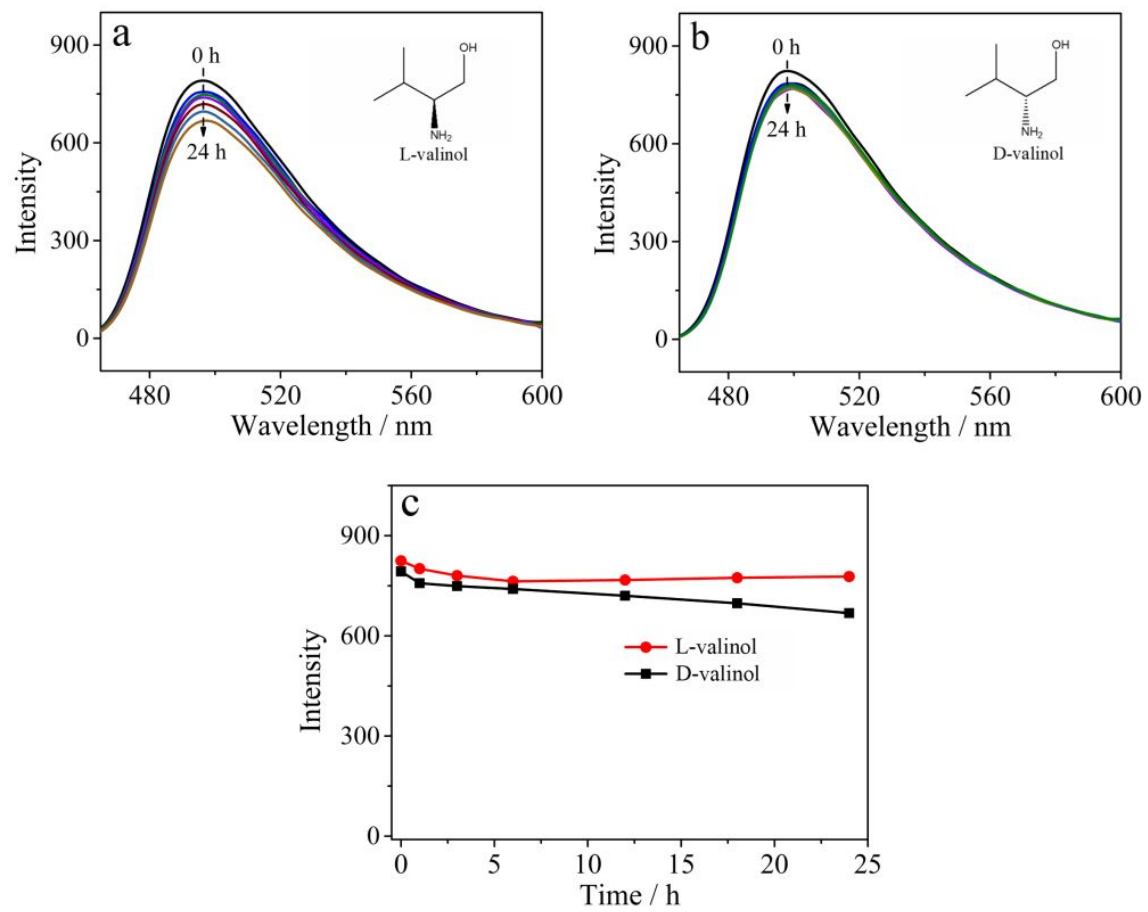

Figure S13. The fluorescence spectra of ThT-LFDF xerogel in the presence of $L$-valinol (a) and $D$ - valinol (b), and the corresponding plots of fluorescence intensity at $488 \mathrm{~nm}$ (c).
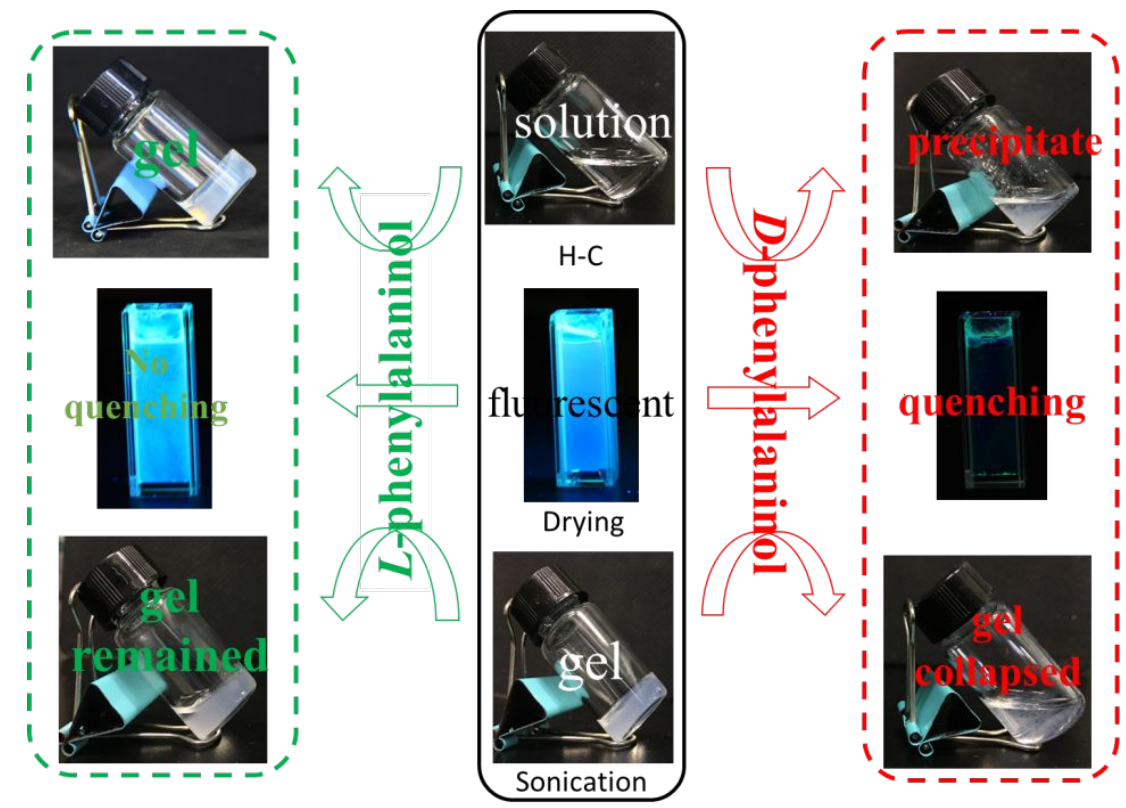

Figure S14. The corresponding discriminate results on L-phenylalaninol (left side) and Dphenylalaninol (right side) through DFLF supramolecular solution (up), supramolecular gel (down) and supramolecular xerogel (middle). 

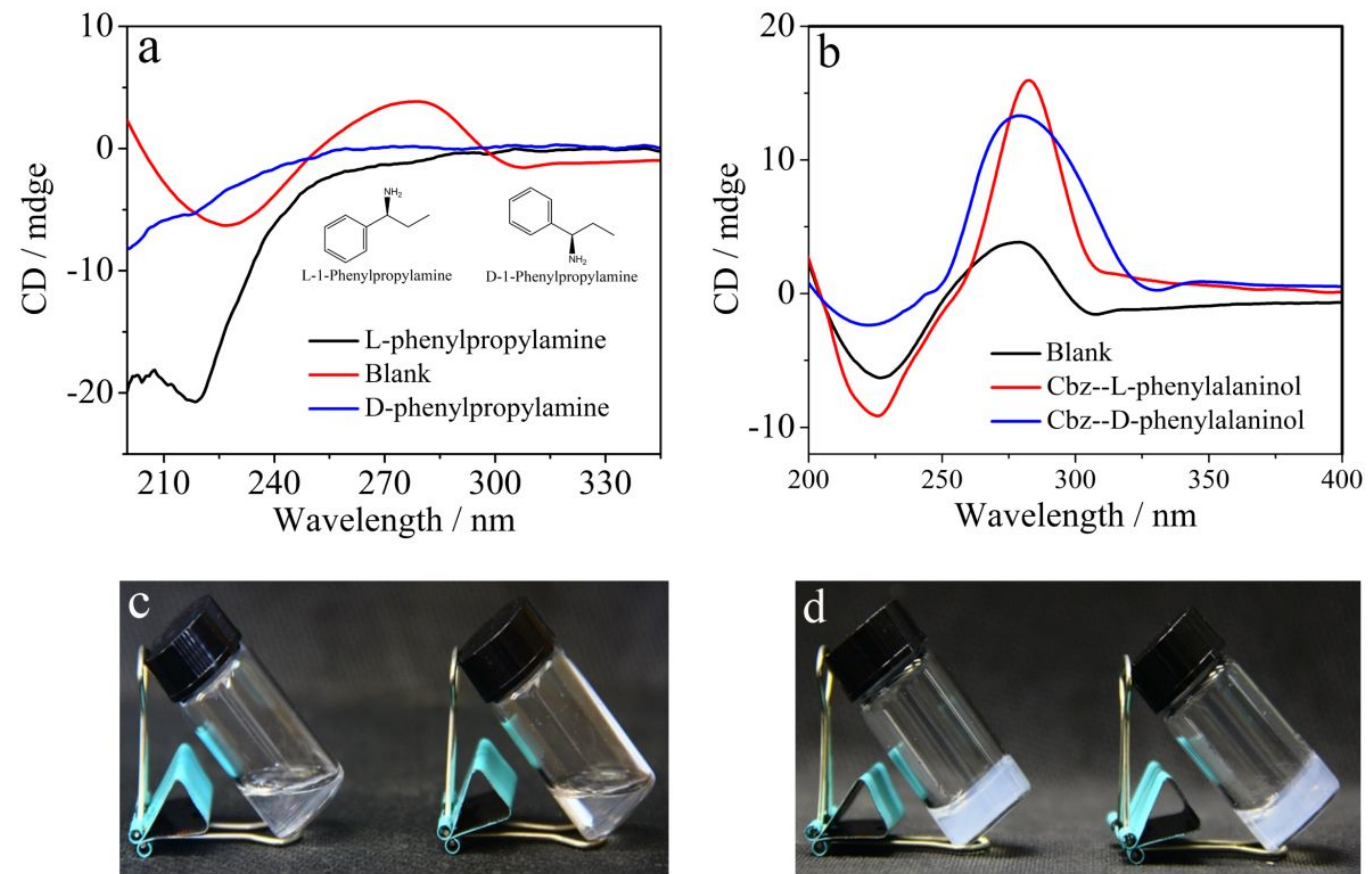

Figure S15. LFDF gel behavior images and CD spectra changes in the presence of phenylalaninol derivatives $L / D$-phenylpropylamine (a) (c) and Cbz- $L / D$-phenylalaninol (b) (d). 